\title{
ScaphoLunate Axis Method
}

\author{
Jeffrey Yao, $\mathrm{MD}^{1}$ Dan A. Zlotolow, MD² Steve K. Lee, MD
}

${ }^{1}$ Department of Orthopaedic Surgery, Stanford University School of Medicine, Redwood City, California

2 Department of Orthopaedic Surgery, Philadelphia Shriners Hospital, Temple School of Medicine, Philadelphia, Pennsylvania

${ }^{3}$ Department of Orthopaedic Surgery, Hospital for Special Surgery, Weill Medical College of Cornell University, New York, New York

Address for correspondence Jeffrey Yao, MD, Department of Orthopaedic Surgery, Stanford University School of Medicine, 450 Broadway Street, Suite C-442, Redwood City, CA 94063 (e-mail: jyao@stanford.edu).

J Wrist Surg 2016;5:59-66.

\begin{abstract}
Keywords

- scapholunate

- ligament

- scapholunate ligament

- scapholunate axis method

Background Treating chronic scapholunate ligament injuries without the presence of arthritis remains an unsolved clinical problem facing wrist surgeons. This article highlights a technique for reconstructing the scapholunate ligament using novel fixation, the ScaphoLunate Axis Method (SLAM).

Materials and Methods In a preliminary review of the early experience of this technique, 13 patients were evaluated following scapholunate ligament reconstruction utilizing the SLAM technique.

Description of Techinque The scapholunate interval is reconstructed utilizing a palmaris longus autograft passed between the scaphoid and lunate along the axis of rotation in the sagittal plane. It is secured in the lunate using a graft anchor and in the scaphoid utilizing an interference screw. The remaining graft is passed dorsally to reconstruct the dorsal scapholunate ligament.

Results At an average follow-up of 11 months, the mean postoperative scapholunate gap was $2.1 \mathrm{~mm}$. The mean postoperative scapholunate angle was 59 degrees. The mean postoperative wrist flexion and extension was 45 and 56 degrees, respectively. The mean grip strength was $24.9 \mathrm{~kg}$, or $62 \%$ of the contralateral side. The mean pain score (VAS) was 1.7. There was 1 failure with recurrence of the pathologic scapholunate gap and the onset of pain.

Conclusion While chronic scapholunate ligament instability remains an unsolved problem facing wrist surgeons, newer techniques are directed toward restoring the normal relationships of the scaphoid and lunate in both the coronal and sagittal planes. The SLAM technique has demonstrated promise in preliminary clinical studies.
\end{abstract}

\section{Rationale and Basic Science}

Chronic scapholunate interosseous ligament (SLIL) deficiency remains an unsolved problem in hand surgery. Scapholunate injuries are the most common intercarpal ligament injury of the wrist, and can be categorized according to severity as predynamic, dynamic, static reducible, static irreducible, or arthritic. $^{1}$ The natural history following SLIL ruptures is

received

June 1, 2015

accepted

December 1, 2015

published online

January 6, 2016 progression to carpal instability with a predictable pattern of degenerative osteoarthrosis of the wrist, known as scapholunate advanced collapse. ${ }^{2}$ When faced with a complete ligament injury, nonsurgical measures are not likely to prevent this progression and thus surgery is required. Surgical treatment is directed toward restoring carpal alignment and kinematics, with the ultimate goals of pain relief and avoidance of the progression to degenerative osteoarthrosis.
Copyright $\odot 2016$ by Thieme Medical Publishers, Inc., 333 Seventh Avenue, New York, NY 10001, USA. Tel: +1(212) 584-4662.
DOI http://dx.doi.org/ 10.1055/s-0035-1570744. ISSN 2163-3916. 
Surgical options include soft-tissue and bony procedures, with SLIL repair for acute injuries ${ }^{3-5}$ and reconstruction or salvage procedures for chronic injuries.

Chronic loss of the SLIL results in dorsal proximal pole scaphoid subluxation, with flexion of the scaphoid about the radioscaphocapitate ligament (RSCL) and pronation along the curve of the capitate. Over time, the RSCL, dorsal intercarpal ligament, and other secondary supporting ligaments stretch to accommodate the new resultant forces. Eventually, the ligamentous and bony changes preclude a closed or even an open reduction. Irreducible scapholunate subluxations are not suitable for soft-tissue reconstructions and are best treated with a salvage procedure.

The best candidates for soft-tissue reconstructions are the subacute or early chronic SLIL disruptions, ideally where the secondary restraints have not been compromised. The Brunelli tenodesis and its modifications attempt to correct the flexion posture and the dissociation of the scaphoid by suspending the scaphoid along a tendon graft connecting the volar trapezium to the dorsal lunate. The tendon lies approximately in line with the normal long axis of the scaphoid. As with all tendon-to-ligament reconstructions, early creep and delayed elongation can be expected proportional to the length of the tendon between fixation points.

Capsulodeses only focus on preventing scaphoid flexion by tethering the distal pole of the scaphoid to either the triquetrum or the distal radius. An additional technique may be required to address the pronation and diastasis of the scaphoid and the dorsal angulation of the lunate.

Unfortunately, current reconstructive and salvage options for chronic injuries are not ideal in restoring carpal kinematics back to the normal state. Current soft-tissue reconstruction procedures do not reliably restore normal carpal alignment and kinematics long term. ${ }^{6-9}$ Additionally, partial wrist arthodeses lead to the immediate alteration of carpal kinematics. 9,10

The rationale for the ScaphoLunate Axis Method (SLAM) is to reconstruct the scapholunate complex with a tendon autograft within the scaphoid and lunate along the central axis of rotation. The graft is fixed close to the scapholunate joint to minimize the effects of creep within the tendon graft due to the short segment of tendon graft between the bones. The idea of a construct placed in the middle of the two bones close the axis of rotation is similar to the RASL (reduction association scaphoid and lunate), initially introduced by Rosenwasser and colleagues. ${ }^{8}$ The tendon graft acts as a flexible biological tether between the two bones rather than a stiff metal screw. The tendon graft that is fixed to bones on either side of a joint may also be compared with the clinically successful reconstruction of the anterior cruciate ligament of the knee. The remaining autograft is passed and secured dorsally to the lunate to reconstruct the important dorsal portion of the scapholunate ligament.

\section{Indications}

The indications for the SLAM technique are still evolving, but it will most likely have the greatest utility in a relatively broad spectrum of pathology, from acute (under 2 weeks), to subacute (between 2 weeks and 3 months), to chronic (over 3 months) stages of scapholunate dissociation. It may be used for dynamic (seen only on radiographic stress views such as the clench pencil view) ${ }^{11}$ or static (seen on nonstress views) cases of dissociation. The scapholunate joint must be reducible (with or without the use of 0.062 -inch K-wires), and there should be no evidence of posttraumatic arthrosis in the radioscaphoid, midcarpal, or radiolunate joints. Reducible is defined as being able to achieve anatomic carpal position with a combination of compressing the scapholunate joint with the c-shaped guide and pushing the capitate head palmarly. If 0.062 -inch K-wires are used in the scaphoid and lunate as reduction joysticks, they should not bend when the bones are reduced. Otherwise, this is too much force and the scapholunate dissociation is considered irreducible. In this scenario, this reconstructive method should not be employed, and a salvage procedure such as a scaphoid excision and fourcorner arthrodesis or proximal row carpectomy is more likely to provide more predictable results. Given the size of drill holes and implants, this reconstruction should only be used in skeletally mature adults with a normal-sized scaphoid and lunate.

\section{Contraindications}

The main contraindications for the SLAM would be an irreducible scapholunate dissociation (unable to reduce with 0.062-inch K-wires without bending them), any evidence of posttraumatic arthrosis in the radioscaphoid, midcarpal, or radiolunate joints, and previous scapholunate repair or reconstruction because of the risk of carpal fracture or ischemia. Similarly, patients with smaller scaphoids or lunates seen on radiographs should not be treated with this technique for fear of fracturing one of the bones.

\section{Surgical Technique for the ScaphoLunate Axis Method}

A dorsal approach to the carpus is made via any type of capsulotomy of the surgeon's preference. A separate $3-\mathrm{cm}$ longitudinal incision is made over the anatomic snuffbox. Lastly, the standard transverse incisions overlying the palmaris longus tendon for harvest of the autograft are used. The vital structures are identified and retracted. These structures include the superficial radial nerve and the radial artery. A capsulotomy is performed to visualize the radial aspect of the scaphoid. The dorsal and palmar aspects of the scaphoid are identified.

The SLAM reconstruction is performed with a kit ( - Fig. 1; Arthrex, Inc., Naples, FL). This kit includes guidewires, a cannulated step drill $(2.85 \mathrm{~mm}$ leading and $3.78 \mathrm{~mm}$ trailing), the tendon graft anchor ("Bullet Anchor," $3.5 \mathrm{~mm} \times 7 \mathrm{~mm}$; - Fig. 2), tendon passer, and the PEEK (polyether ether ketone) scaphoid interference screw (4 mm $\times 10 \mathrm{~mm}$ ). A c-shaped reduction drill guide is very useful for maintaining the reduction as well as guiding the placement of the central guidewire. This reusable guide is packaged separately. 


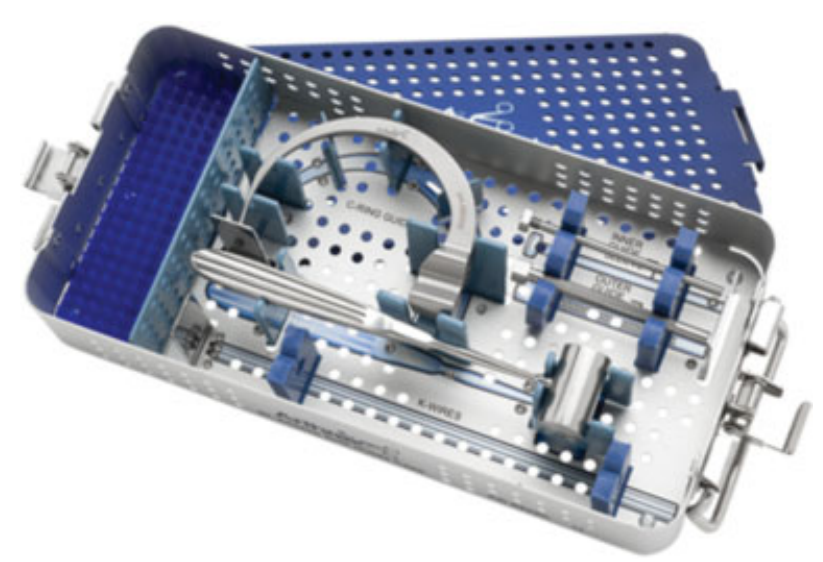

Fig. 1 The SLAM kit. (Courtesy of Arthrex, Inc.)

The pointed tip of the c-shaped reduction guide is placed on the proximal ulnar aspect of the lunate at its midpoint in the anteroposterior dimension. Access to this spot is achieved by making a transverse incision in the capsular tissue ulnar to the dorsal radiocarpal (DRC) ligament. Alternatively, extending the inverted T-shaped capsulotomy as far ulnarly as possible aids in visualization of proximal ulnar corner of the lunate. Separate dissection and retraction of the extensor digiti minimi is usually needed. The cannulated compression sleeve of the reduction guide is then applied to the scaphoid. It is important for the starting point on the scaphoid to be midlateral between the dorsal and palmar borders. The scapholunate joint is then reduced by compressing the joint with the ratcheting mechanism of the c-shaped reduction drill guide while at the same time pushing the capitate head palmarly. When the capitate head is pushed palmarly, the lunate is reduced out of dorsal intercalated segmental instability (DISI; - Fig. 3). An axial load of the carpus can help "mold" the scapholunate joint to the capitate head. Reduction occasionally requires the use of joystick, 0.062-inch K-wires, placed in the lunate and the distal pole of the scaphoid from

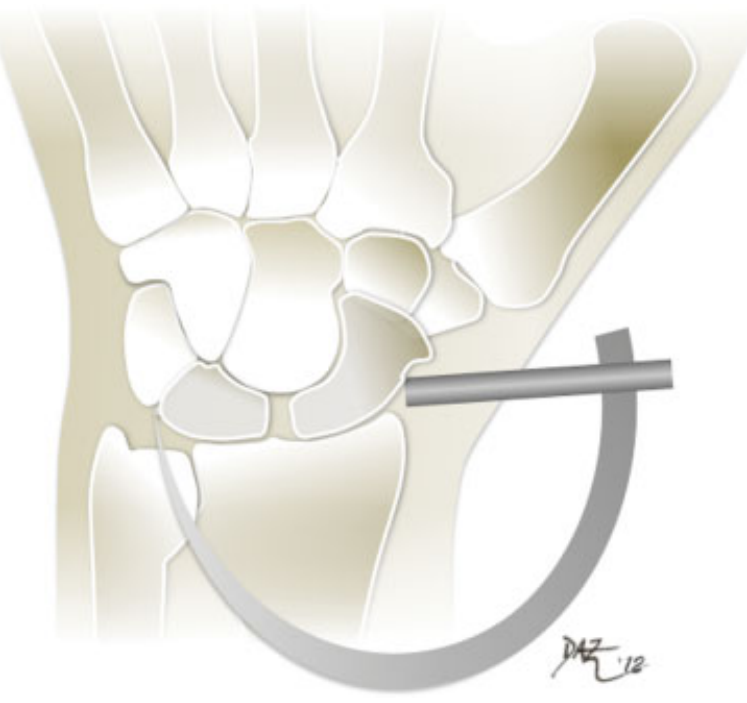

Fig. 3 Placement of the reduction c-ring guide. (Courtesy of Dan A. Zlotolow, MD.)

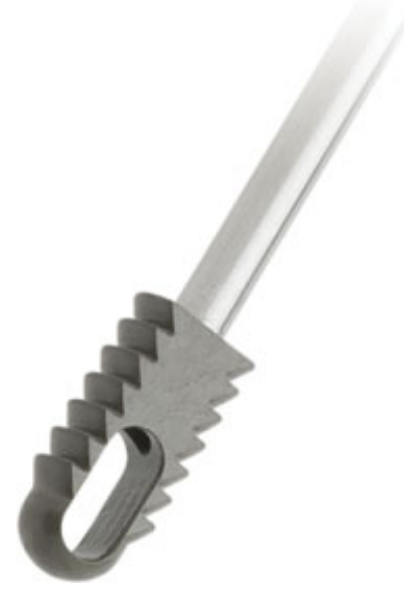

Fig. 2 The graft anchor. (Courtesy of Arthrex, Inc.)

dorsal to palmar. Once the scapholunate joint is reduced, it is pinned with the central guidewire through the inner sleeve on the c-shaped reduction drill guide. The guidewire must be in the center of the scaphoid and lunate in the sagittal plane and in the proximal ulnar corner of the lunate in the coronal plane. This is the most essential portion of this procedure and great care must be taken to ensure the correct position of this guidewire. Eccentric placement outside of the center of the scaphoid and lunate in the lateral plane may lead to disruption of the cortices of the scaphoid or lunate during the subsequent steps and should not be tolerated. A secondary wire is used to stabilize the carpus and to assure that the reduction guide stays in place during the remainder of the procedure (-Fig. 4A, B). The distal palmar hole adjacent to the central wire on the guide is frequently the best to use for the secondary wire. Anatomic reduction and precise pin placement is confirmed on posteroanterior (PA) and lateral fluoroscopic images. The radio-opaque drill guide will often obstruct the fluoroscopic image. Instead of trying to move the guide and thus stressing the construct, angling the arm of the guide a few degrees distally or proximally is sufficient to move the opaque guide out of the way of the X-ray beam. The optimal position of the tip of the central wire is at the proximal ulnar corner of the lunate on the PA view and central within the scaphoid and lunate on the lateral view. The wire is passed across the scapholunate joint slightly distal to its midaspect. The cannulated step drill (distal end has a narrower diameter than proximal end) is then drilled over the central guidewire ( $\mathbf{- F i g . 5 A , B}$ ) and stopped before reaching the far cortex of the lunate. The drill hole in the lunate is 10.7 $\mathrm{mm}^{2}$ and the mean surface area of the lunate joint surface with the scaphoid is $190 \mathrm{~mm}^{2}$. The percentage of the drill hole to the joint surface is therefore $\sim 5.6 \%$. The depth of the drill is measured off the c-clamp guide and confirmed under fluoroscopy. The holes in the scaphoid and lunate are irrigated with normal saline with a long spinal needle and syringe to remove the debris that may block easy passage of the tendon graft. The tendon autograft (palmaris longus or alternatively one-half of flexor carpi radialis or other tendon autograft) is shuttled into the lunate anchor through the eyelet with the 
A

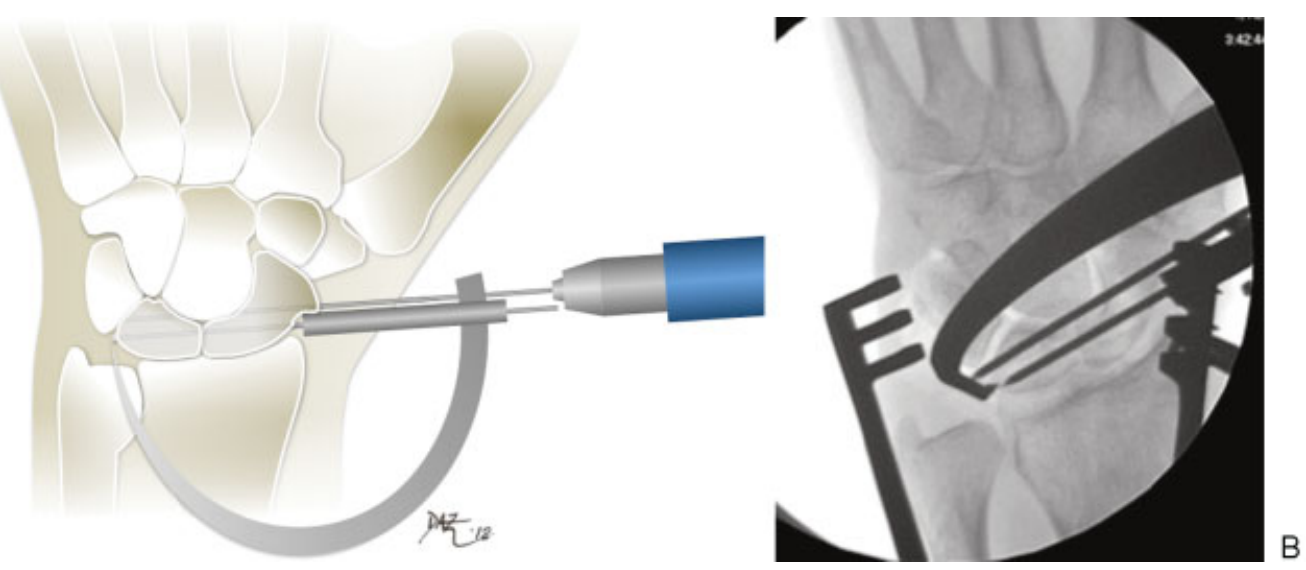

Fig. 4 (A) Placement of the central guidewire in the perfect center-center position in the lunate and scaphoid. Placement of the secondary stabilization wire as well. (Courtesy of Dan A. Zlotolow, MD.) (B) Radiograph (wrist PA) of two wires placed.

A

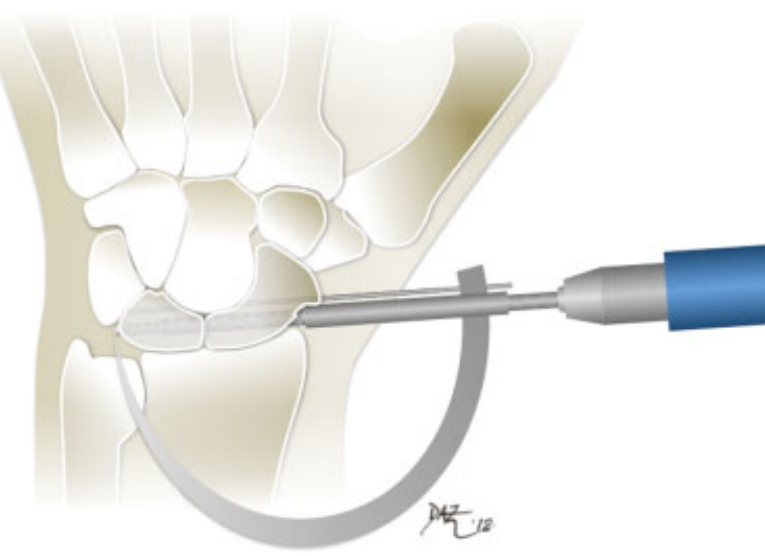

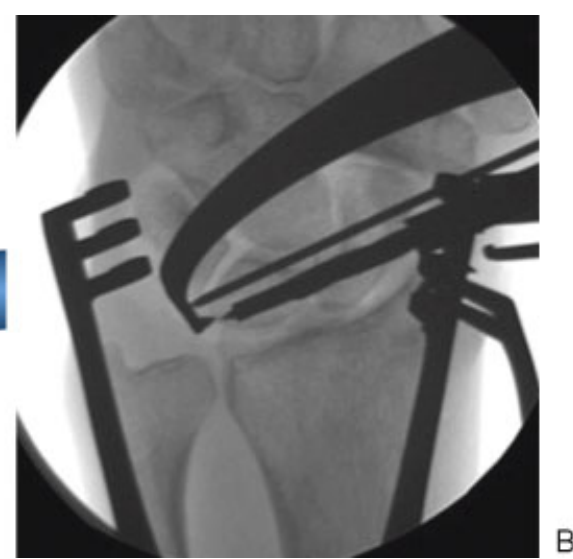

B

Fig. 5 (A) Passing the step drill into the lunate over the central guidewire. (Courtesy of Dan A. Zlotolow, MD.) (B) Radiograph (wrist PA) of step drill in place.

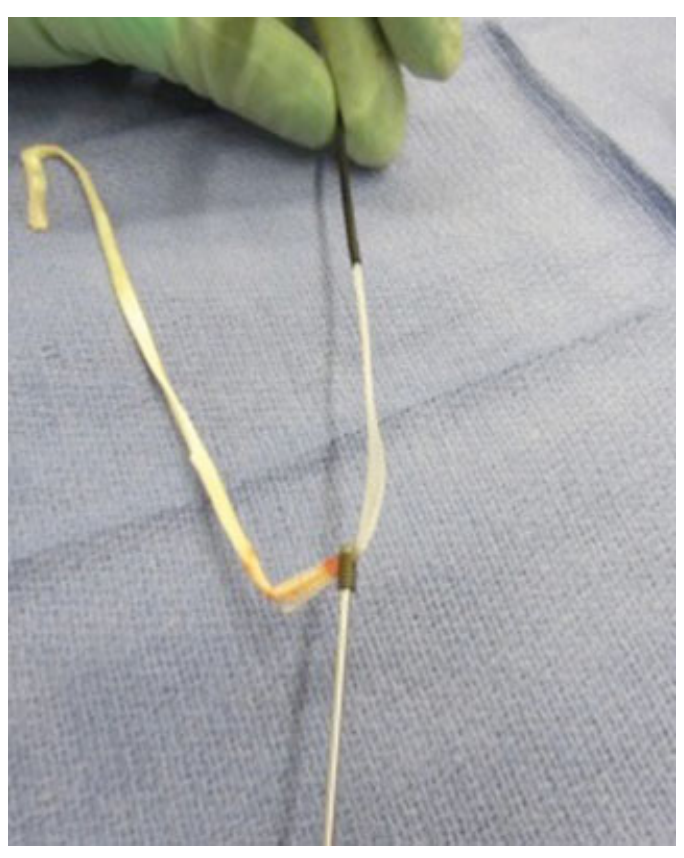

Fig. 6 Tendon graft threaded through the anchor. tendon passer (-Fig. 6). The tendon graft is dressed on the lunate bullet anchor by pulling it taut on the bullet anchor inserter. Care is taken to make sure that the tendon does not cover the reverse barbs on the bullet anchor. The anchor with tendon autograft is passed through the outer sleeve of the reduction guide. If it does not pass easily, the tendon graft must be trimmed or it will not pass into the lunate. The

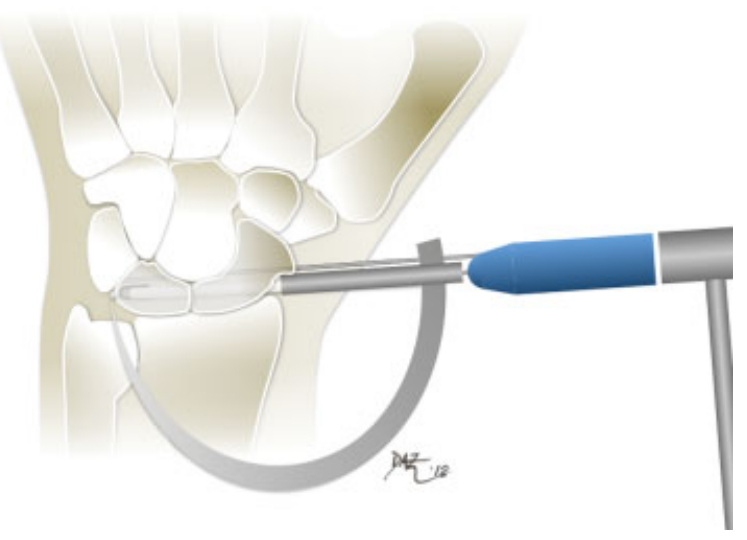

Fig. 7 Impacting the anchor with the tendon graft into the lunate. (Courtesy of Dan A. Zlotolow, MD.) 


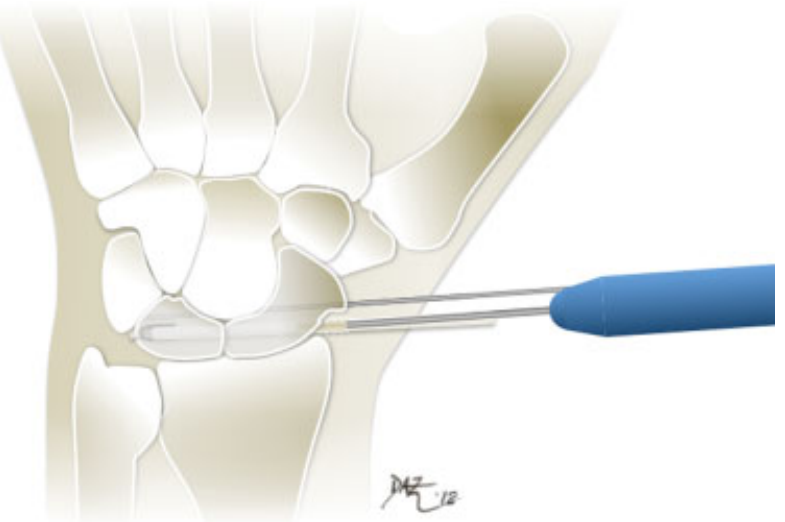

Fig. 8 Placement of the PEEK interference screw into the scaphoid. (Courtesy of Dan A. Zlotolow, MD.)

anchor with the tendon is then advanced until mild resistance is encountered, typically at the point where the narrower hole in the lunate starts. Using a mallet, the anchor and graft are tapped until seated into the ulnar side of the lunate under fluoroscopic guidance ( - Fig. 7). The second wire is left in for 6 to 8 weeks.

Biomechanical testing has shown pullout strength of the anchor and tendon graft in a Sawbones model (Model \#152204, Pacific Research Laboratories, Vashon, WA) to be $161.5 \mathrm{~N}$. The density of these blocks is comparable to that of normal human cancellous bone (30 lb/ $\mathrm{ft}^{3}$; unpublished internal data, Sawbones, Pacific Research Laboratories, Vashon, WA). The final position of the anchor should be near the proximal ulnar corner of the lunate. While holding tension on both free tendon ends to pretension the graft, the scaphoid interference screw is then placed into the hole in the scaphoid alongside the tendon graft parallel to the second wire (-Fig. 8) until the screw is buried beneath the radial cortex of the scaphoid (-Fig. 9). The excess tendon graft is then passed radially and dorsally over the carpus and fixed to the dorsal aspect of the lunate. The tendons are sutured to the remnant of the scapholunate ligament if available, then to the

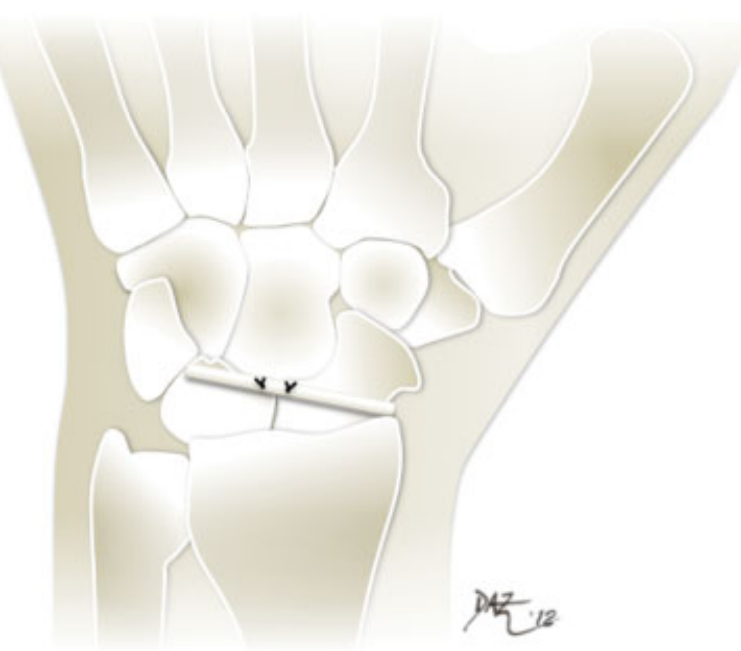

Fig. 10 Final reconstruction with dorsal stabilization of the remainder of the graft. (Courtesy of Dan A. Zlotolow, MD.)

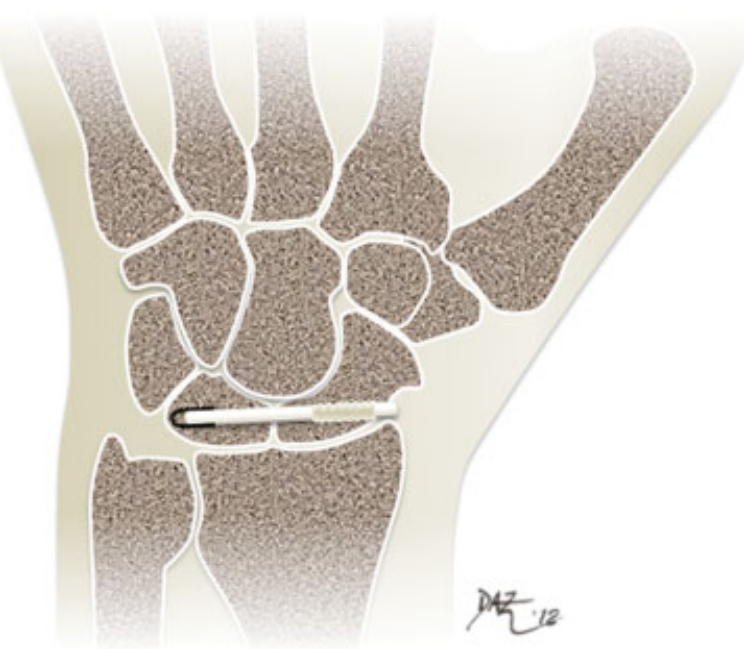

Fig. 9 Final reconstruction (coronal cut through the carpus). (Courtesy of Dan A. Zlotolow, MD.)

DRC ligament as it attaches to the triquetrum and/or the lunotriquetral ligament (-Fig. 10). The dorsal aspects of the lunate and scaphoid that the graft passes above are decorticated to provide a healthy bleeding bony bed to allow for healing of the graft.

\section{Alternative Surgical Technique}

If the surgeon does not wish to use the c-clamp targeting guide to drill the path for the graft in one pass, an alternative technique may be employed. This technique involves drilling the lunate and scaphoid independently in two separate steps. Because the autograft used for the reconstruction is pliable, the tunnels in the scaphoid and lunate may not need to be perfectly collinear as would be obtained with a single drill technique as described above. Therefore, some surgeons may find this alternative technique somewhat simpler than the single drill technique.

The lunate and scaphoid are drilled independently. The scapholunate interval is opened to expose the radial articular

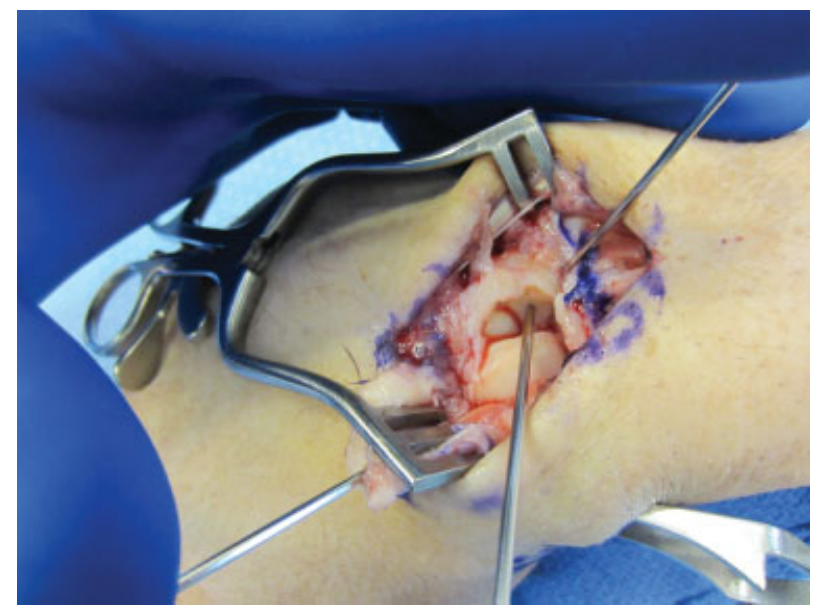

Fig. 11 The double-drill technique. The first guidewire is placed into the center of the articular surface of the lunate advancing it toward the proximal ulnar corner of the lunate. 


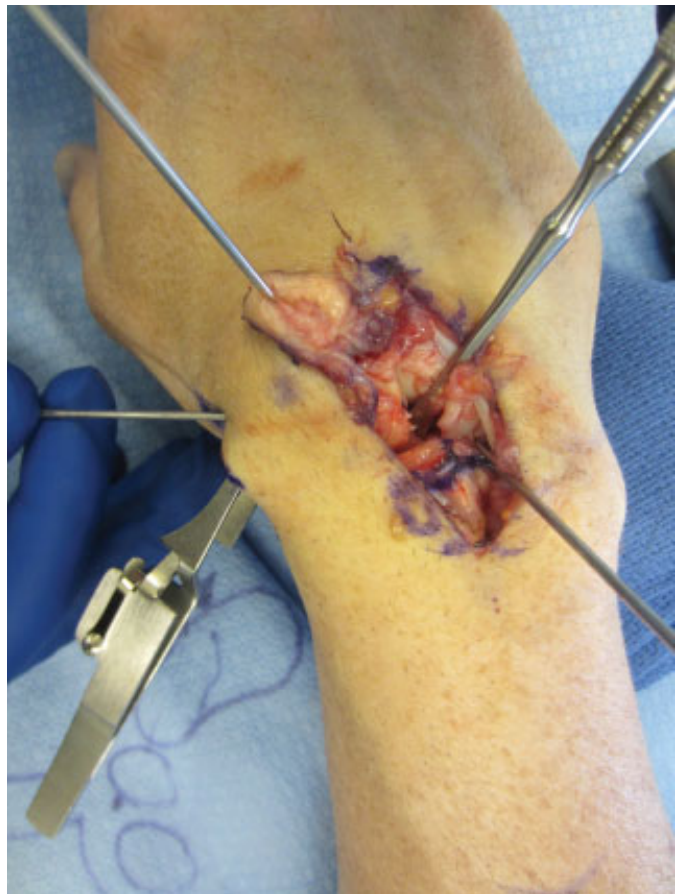

Fig. 12 The double-drill technique. The second guidewire is placed into the center of the articular surface of the scaphoid advancing it to the ridge between the articular and nonarticular borders of the scaphoid.

surface of the lunate and the ulnar articular surface of the scaphoid. The first guidewire is drilled into the lunate in the center of the radial articular surface in a trajectory toward the proximal ulnar corner of the lunate (-Fig. 11). Its appropriate position is confirmed on fluoroscopy, and the cannulated step drill is then used to drill the lunate alone until the drill approaches the ulnar cortex. Once the lunate tunnel is drilled and confirmed under fluoroscopy, the guidewire is removed and a new guidewire is placed in the ulnar articular surface of the scaphoid in a trajectory toward the waist of the scaphoid. This wire is drilled through the radial cortex of the scaphoid and exits the radial wound, while the branches of the dorsal radial sensory nerve and dorsal branch of the radial artery are protected (-Fig. 12). Once the correct position of the wire is confirmed under fluoroscopy, the scaphoid is drilled with the step drill, ensuring the entire scaphoid is drilled with the trailing threads of the cannulated drill. Once both tunnels are confirmed, the graft is prepared as described above and first impacted into the lunate through the SL interval (-Fig. 13). Once the proper position of the graft is confirmed fluoroscopically, the handle of the inserter is removed and the two tails of the graft are passed through the scaphoid from the ulnar side out through the radial incision (-Fig. 14). The interference screw is placed and the remaining steps are completed as described above. Use of a large point reduction clamp to compress the scapholunate joint can be considered.

\section{Rehabilitation}

The patient is placed in a short arm thumb spica splint for 2 weeks postoperatively. At 2 weeks, the sutures are removed and a short arm cast is applied. After 6 weeks, the cast and the

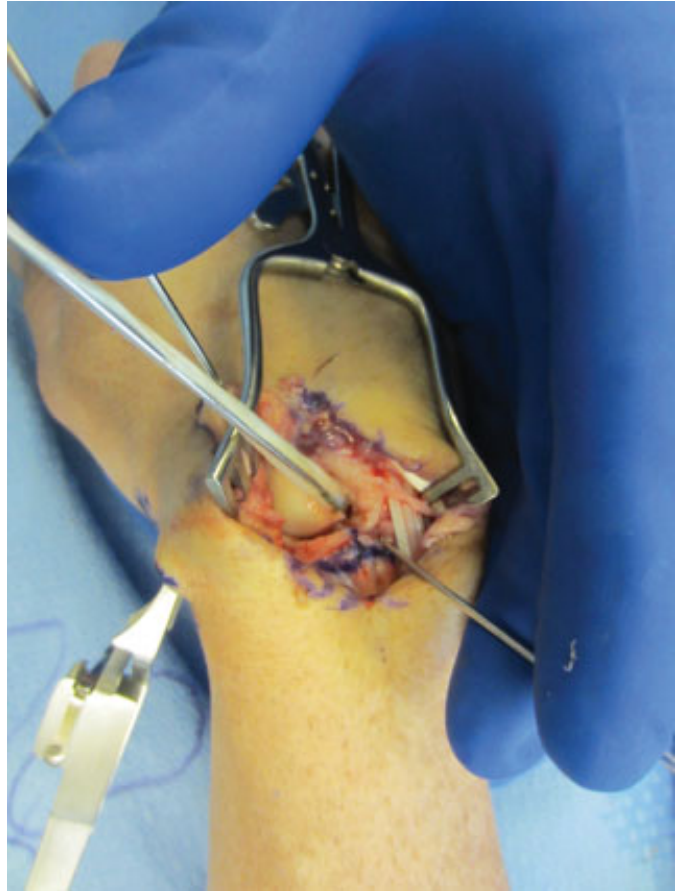

Fig. 13 The double-drill technique. The graft anchor and graft are impacted into the lunate.

second K-wire are removed, and the patient is placed in a thermoplastic wrist splint in slight extension (15 degrees) made by the hand therapist. Gentle active and active-assisted range of motion of the wrist is initiated. Initially, dart throwers motion $^{8}$ and supination/pronation are performed, then wrist flexion, extension, and radial and ulnar deviation at 8 to 9 weeks. Strengthening is started 10 to 12 weeks postoperatively. Light weight lifting and sports may start at 4 months postoperatively. No contact sports are allowed for 6 to 9 months, depending on the sport and level of activity. Activity modification of avoiding extended wrist loading is discussed with the patient. Loading should be with a neutral wrist, as this minimizes the strain on the scapholunate ligament complex.

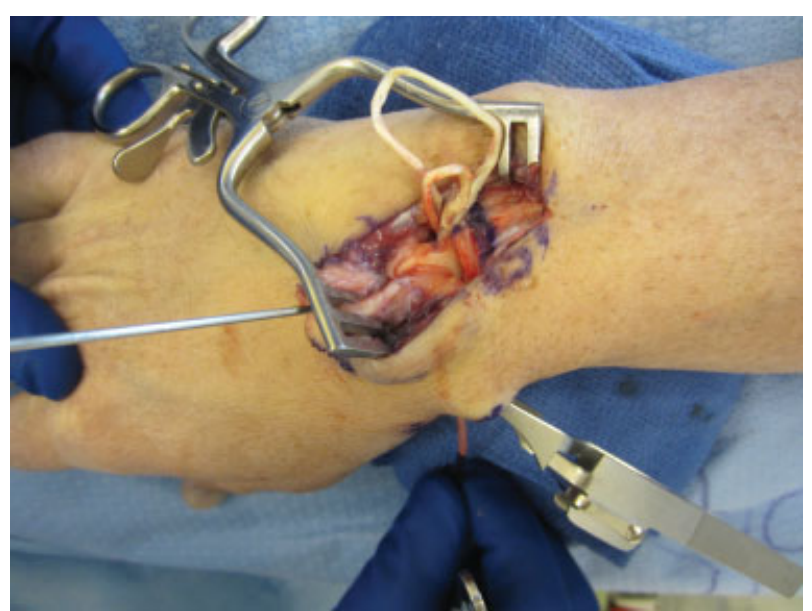

Fig. 14 The double-drill technique. The two tails of the graft are passed through the hole in the scaphoid via a suture passer (seen at the bottom of the figure). 


\section{Potential Complications}

In addition to standard potential surgical complications, the specific potential risks of this procedure are carpal fracture, carpal ischemia, graft or implant failure, and loss of reduction. Carpal fracture is the most serious possible complication, but is avoidable if adherence to the described surgical technique is ensured. Namely, the most important step of the technique is the placement of the initial guidewire exactly in the center of the scaphoid and lunate on the lateral view. If the guidewire is central, the subsequent drill hole and implant/graft will be central. Eccentric placement of the wire, either dorsally or palmarly, puts the scaphoid or lunate at risk for fracture when drilling, inserting the graft and anchor, and inserting the interference screw in the scaphoid (secondary to hoop stresses). If the surgeon finds that the scaphoid hole and implant/graft are too dorsal or palmar, the interference screw should be abandoned and the tendon ends are pulled taut and tied dorsoulnarly to the DRC ligament and/or lunotriquetral ligament. On the PA view, the best line of placement of the graft is just distal to the midpoint of the scapholunate joint.

\section{Preliminary Results}

At the time of this writing, there have been $\sim 45$ cases of SLAM performed for scapholunate instability in the United States and an additional 70 cases in Europe. The preliminary reports from surgeons have been generally favorable, and clinical data following at least 2 years of follow up are currently being collected. In a preliminary review of the early experience of this technique for three academic hand surgeons in the United States, the outcomes of 13 patients treated since 2012 were evaluated (unpublished data). For this cohort, the mean follow-up period was 11 months (range: 3-25). The average age of the patients was 48 years (range: 29-65). The mean preoperative scapholunate gap was $5.4 \mathrm{~mm}$ and the mean postoperative gap was $2.1 \mathrm{~mm}(p<0.05)$. The mean preoperative and postoperative scapholunate angle was 70 and 59 degrees, respectively $(p<0.05)$. The mean postoperative wrist flexion and extension was 45 and 56 degrees, respectively. The mean grip strength was $24.9 \mathrm{~kg}$, or $62 \%$ of the contralateral side. The mean visual analog scale for pain was 1.7 (range: $0-4$ ). There was one failure with restoration of the pathologic scapholunate gap and the onset of pain. This patient was the oldest of the cohort (65 years) and was ultimately treated with a proximal row carpectomy with good short-term results. Arguably, the age of this patient was a relative contraindication for a reconstructive procedure, and a salvage procedure should have been considered initially.

\section{Discussion}

Surgical methods of treating scapholunate instability include direct repair, capsulodeses, tendon reconstructions, tenodeses, bone-ligament-bone reconstruction, scapholunate fusion, scapholunate screw, and others. $2,5,6,8,12-17$ No reconstruction to date has reliably restored scaphoid exten- sion, closed the SL gap, and corrected scaphoid pronation. The ideal scapholunate reconstruction should (1) provide a biologic solution for a biologic (ligament injury) problem, (2) reconstruct both the volar and dorsal SLIL to avoid eccentric gapping, (3) place fixation points close to the SL joint to minimize creep, (4) avoid having tissue or implants on articular surfaces, (5) allow for eventual all arthroscopic reconstruction, and (6) restore normal kinematics to both optimize motion and prevent late degenerative changes.

Several capsulodesis techniques have been used for the treatment of these injuries. Although they generally address scaphoid flexion in the sagittal plane, they do not reliably reduce and stabilize the scapholunate gap in the coronal plane. ${ }^{6,17}$ Not associating the scaphoid and the lunate allows the lunate to remain dorsiflexed, leading to abnormal loading patterns. ${ }^{8}$ While the results of capsulodesis have not been tied to maintenance of a reduced scapholunate interval, the number of patients included in these studies and their relative heterogeneity make it difficult to conclude that associating the scaphoid and the lunate does not matter. Furthermore, the procedures that tether the scaphoid to the distal radius may limit wrist flexion.

Several tenodesis procedures have been used as an alternative to capsulodesis to restore carpal stability while preserving motion. Some techniques tether the scaphoid to the distal radius limiting wrist flexion. ${ }^{18}$ These reconstructive procedures also focus on reconstructing the dorsal scapholunate ligament only. Reconstructing the dorsal ligament only may lead to gapping of the volar interval, also leading to abnormal kinematics and loading. Overall, favorable shortterm results have been reported for less severe injuries, but long-term studies are lacking. ${ }^{12,18-20}$ There has not been any proven benefit of tenodesis versus capsulodesis. ${ }^{21}$

Scapho-trapezio-trapezoid arthrodesis has been used as an alternative method of treatment for this condition. Carpal kinematics and motion are altered as the scaphoid is linked to the distal carpal row, and long-term studies have shown up to a $50 \%$ progression to arthrosis. ${ }^{10,22}$

Scapholunate arthrodesis has also been described in the treatment of scapholunate dissociation. The procedure is associated with a high rate of nonunion, although several authors have reported favorable clinical results with a fibrous nonunion. ${ }^{15,23-25}$ In a modification of Herbert scapholunate fusion, the reduction and association of the scaphoid and lunate (RASL) procedure was developed to ideally create a fibrous nonunion between the scaphoid and lunate. ${ }^{8,16}$ Since the SL axis is inconstant during wrist motion, ${ }^{26}$ the screw tends to spin eccentrically within the scaphoid and/or the lunate which may eventually lead to cavitary bone loss and screw loosening. Some surgeons routinely remove the screw to prevent this complication. To date, there are limited outcomes data.

This manuscript describes a technique for SL reconstruction which utilizes a two-tailed tendon autograft placed along the axis of rotation of the scaphoid and lunate and secured using a tendon graft anchor. This is coupled with reconstruction of the critical dorsal SL ligament. The SLAM leads to improved SL interval and SL angle correction when compared 
with current techniques of SL reconstruction. ${ }^{27,28}$ While better radiographic parameters do not necessarily portend a favorable clinical outcome, the results are encouraging that the concept is biomechanically sound.

The goals of the SLAM reconstruction are to create a multiplanar tether between the scaphoid and lunate bones. The reconstruction does not restore volar SLIL function. Instead, one of the tethers is placed in the central axis between the scaphoid and lunate bones, borrowing on the promising data from the RASL reconstruction. The SLAM technique also incorporates a dorsal tendon graft reconstruction, which recreates the dorsal portion of the SLIL. The dorsal SLIL secures the scaphoid from subluxating dorsally out of the lip of the dorsal radius and resists scaphoid flexion and pronation. Together, the double tether may help to prevent scapholunate translation and diastasis better than a single spanning reconstruction.

Because there is not one true axis between the scaphoid and the lunate throughout wrist motion in any plane, the SLAM has at least a theoretical advantage over the RASL in that it is a biological repair and that the tendon graft is not as rigid a construct as a metal screw. Another possible advantage over previous techniques is that fixation of the autograft is achieved near the SL joint in both the lunate and scaphoid, reducing the potential for creep compared with previous methods.

The SLAM reconstruction holds promise for exceeding the biomechanical characteristics of two of the common procedures performed for scapholunate ligament reconstruction (Blatt capsulodesis and modified Brunelli 3-ligament tenodesis). ${ }^{28}$ The initial clinical results with this technique are favorable, but the caveat is that the studies of the longterm outcomes of this procedure are currently lacking, and enthusiasm for this technique should be tempered until these studies are performed. Currently, clinical data of patients who have had this procedure done at least 2 years ago are being collected in the United States and Europe.

\section{Conflict of Interest}

None.

\section{References}

1 Kuo CE, Wolfe SW. Scapholunate instability: current concepts in diagnosis and management. J Hand Surg Am 2008;33(6):998-1013

2 Watson HK, Ballet FL. The SLAC wrist: scapholunate advanced collapse pattern of degenerative arthritis. J Hand Surg Am 1984; 9(3):358-365

3 Palmer AK, Dobyns JH, Linscheid RL. Management of post-traumatic instability of the wrist secondary to ligament rupture. J Hand Surg Am 1978;3(6):507-532

4 Conyers DJ. Scapholunate interosseous reconstruction and imbrication of palmar ligaments. J Hand Surg Am 1990;15(5):690-700

5 Lavernia CJ, Cohen MS, Taleisnik J. Treatment of scapholunate dissociation by ligamentous repair and capsulodesis. J Hand Surg Am 1992;17(2):354-359
6 Gajendran VK, Peterson B, Slater RR Jr, Szabo RM. Long-term outcomes of dorsal intercarpal ligament capsulodesis for chronic scapholunate dissociation. J Hand Surg Am 2007;32(9):1323-1333

7 Glickel SZ, Millender LH. Ligamentous reconstruction for chronic intercarpal instability. J Hand Surg Am 1984;9(4):514-527

8 Rosenwasser MP, Miyasajsa KC, Strauch RJ. The RASL procedure: reduction and association of the scaphoid and lunate using the Herbert screw. Tech Hand Up Extrem Surg 1997;1(4):263-272

9 Bloom HT, Freeland AE, Bowen V, Mrkonjic L. The treatment of chronic scapholunate dissociation: an evidence-based assessment of the literature. Orthopedics 2003;26(2):195-203, quiz 204-205

10 Kleinman WB, Carroll C IV. Scapho-trapezio-trapezoid arthrodesis for treatment of chronic static and dynamic scapho-lunate instability: a 10-year perspective on pitfalls and complications. J Hand Surg Am 1990;15(3):408-414

11 Lee SK, Desai H, Silver B, Dhaliwal G, Paksima N. Comparison of radiographic stress views for scapholunate dynamic instability in a cadaver model. J Hand Surg Am 2011;36(7):1149-1157

12 Garcia-Elias M, Lluch AL, Stanley JK. Three-ligament tenodesis for the treatment of scapholunate dissociation: indications and surgical technique. J Hand Surg Am 2006;31(1):125-134

13 Blatt G. Capsulodesis in reconstructive hand surgery. Dorsal capsulodesis for the unstable scaphoid and volar capsulodesis following excision of the distal ulna. Hand Clin 1987;3(1):81-102

14 Weiss AP. Scapholunate ligament reconstruction using a boneretinaculum-bone autograft. J Hand Surg Am 1998;23(2):205-215

15 Hom S, Ruby LK. Attempted scapholunate arthrodesis for chronic scapholunate dissociation. J Hand Surg Am 1991;16(2):334-339

16 Aviles AJ, Lee SK, Hausman MR. Arthroscopic reduction-association of the scapholunate. Arthroscopy 2007;23(1):105.e1-105.e5

17 Moran SL, Cooney WP, Berger RA, Strickland J. Capsulodesis for the treatment of chronic scapholunate instability. J Hand Surg Am 2005;30(1):16-23

18 Brunelli GA, Brunelli GR. A new technique to correct carpal instability with scaphoid rotary subluxation: a preliminary report. J Hand Surg Am 1995;20(3, Pt 2):S82-S85

19 Chabas JF, Gay A, Valenti D, Guinard D, Legre R. Results of the modified Brunelli tenodesis for treatment of scapholunate instability: a retrospective study of 19 patients. J Hand Surg Am 2008;33(9):1469-1477

20 Van Den Abbeele KL, Loh YC, Stanley JK, Trail IA. Early results of a modified Brunelli procedure for scapholunate instability. J Hand Surg [Br] 1998;23(2):258-261

21 Moran SL, Ford KS, Wulf CA, Cooney WP. Outcomes of dorsal capsulodesis and tenodesis for treatment of scapholunate instability. J Hand Surg Am 2006;31(9):1438-1446

22 Fortin PT, Louis DS. Long-term follow-up of scaphoid-trapeziumtrapezoid arthrodesis. J Hand Surg Am 1993;18(4):675-681

23 Armstrong GW. Rotational subluxation of the scaphoid. Can J Surg 1968;11(3):306-314

24 Hastings DE, Silver RL. Intercarpal arthrodesis in the management of chronic carpal instability after trauma. J Hand Surg Am 1984; 9(6):834-840

25 Russell TB. Inter-carpal dislocations and fracture-dislocations; a review of 59 cases. J Bone Joint Surg Br 1949;31B(4):524-531, illust

26 Crisco JJ, Heard WM, Rich RR, Paller DJ, Wolfe SW. The mechanical axes of the wrist are oriented obliquely to the anatomical axes. J Bone Joint Surg Am 2011;93(2):169-177

27 Pollock PJ, Sieg RN, Baechler MF, Scher D, Zimmerman NB, Dubin $\mathrm{NH}$. Radiographic evaluation of the modified Brunelli technique versus the Blatt capsulodesis for scapholunate dissociation in a cadaver model. J Hand Surg Am 2010;35(10):1589-1598

28 Lee SK, Zlotolow DA, Sapienza A, Karia R, Yao J. Biomechanical comparison of 3 methods of scapholunate ligament reconstruction. J Hand Surg Am 2014;39(4):643-650 